\title{
Growth inhibitory effects of vitamin K2 on colon cancer cell lines via different types of cell death including autophagy and apoptosis
}

\author{
HIDEAKI KAWAKITA ${ }^{1}$, AKIHIKO TSUCHIDA ${ }^{1}$, KEISUKE MIYAZAWA ${ }^{2}$, MUNEKAZU NAITO ${ }^{3}$, \\ MASATOSHI SHIGOKA ${ }^{1}$, BUNSO KYO ${ }^{1}$, MASANOBU ENOMOTO ${ }^{1}$, TATEHIKO WADA ${ }^{1}$, KENJI KATSUMATA ${ }^{1}$, \\ KAZUMA OHYASHIKI ${ }^{4}$, MASAHIRO ITOH ${ }^{3}$, AKIO TOMODA ${ }^{2}$ and TATSUYA AOKI ${ }^{1}$ \\ ${ }^{1}$ Third Department of Surgery, ${ }^{2}$ Department of Biochemistry, ${ }^{3}$ Department of Anatomy, \\ ${ }^{4}$ First Department of Internal Medicine, Tokyo Medical University, Tokyo, Japan
}

Received January 27, 2009; Accepted March 19, 2009

DOI: $10.3892 /$ ijmm_00000184

\begin{abstract}
Vitamin K2 (menaquinone-4: MK4) has been reported to inhibit cell growth and induce apoptosis in various tumor cells. We examined the effects of MK4 using three types of colon cancer cell lines: PMCO1, COLO201, and DLD-1. Exposure to MK4 was at concentrations from 5 to $50 \mu \mathrm{M}$, growth inhibitory effects were observed dose-dependently in COLO201 and PMCO1, whereas the growth inhibition observed in DLD-1 was minimal. Comparison of COLO201 and PMCO1 cells exhibiting distinct growth inhibitory effects showed that cell death via apoptosis accompanied by activation of caspase- 3 was induced in PMCO1, while apoptosis was not induced in COLO201. On the contrary, immunoblot assay using an anti-LC3B antibody showed autophagy induction by addition of MK4 and incubation in all three types of colon cancer cell lines. Addition of 3 methyladenine (3-MA) attenuated the growth inhibitory effect of MK4 in COLO201, whereas no influence of 3-MA was noted in PCMO1. Electron microscopy images of COLO201 showed that addition of MK4 induced an increased number of cytoplasmic autophagosomes and autolysosomes as well as morphological changes including scantiness of cytoplasm accompanied by loss of cell organelles, nuclear shrinkage, and fragmentation of cytoplasmic membrane in some cells, indicating the induction of cell death via autophagy not accompanied by the formation of apoptotic bodies in COLO201 cells. These results suggested that the response to MK4 and the way of induction of cell death vary in different colon cancer cell lines.
\end{abstract}

Correspondence to: Dr Akihiko Tsuchida, Third Department of Surgery, Tokyo Medical University, 6-7-1 Nishi-Shinjuku, Shinjuku-ku, Tokyo 160-0023, Japan

E-mail: akihikot@tokyo-med.ac.jp

Key words: colon cancer, vitamin K2, chemoprevention, autophagy, apoptosis

\section{Introduction}

Vitamin K2 (VK2, or menaquinone) is described as 'menaquinone-n (MKn)' according to the number of isoprenoid radicals that make up its side chain, and a total of 14 types of MKn (MK1-MK14) are found in nature. Among these MKn types, MK4 (menatetrenone), which has a geranylgeraniol radical as its side chain, is not only used for the treatment of diseases, such as osteoporosis and hypoprothrombinemia, but also known to exert in vitro and in vivo anti-tumor effects (1). Clinical investigations on malignant tumors have reported a decreased percentage of blastic cells, an increased number of mature neutrophils, or improvement in anemia or decreased platelet counts in patients with myelodysplastic syndrome (MDS) (2) and a decreased number of blastic cells by oral treatment with MK4 in patients with post-myelodysplastic syndrome AML (3). In addition, it was shown that female patients with viral cirrhosis of the liver in the MK4-treated group had a significantly lower incidence of hepatocellular carcinoma than those in the control group (4). Furthermore, patients with adjuvant treatment of MK4, who had curatively treated hepatocellular carcinoma, had a significantly reduced recurrence rate (5). As described in these reports, since MK4 causes few side effects, thereby allowing long-term oral administration, MK4 may be effective as a chemopreventive agent for cancer.

Although detailed information concerning the mechanisms of the cell growth inhibitory effects of MK4 are not completely understood, it is evident from our previous in vitro studies that the effect is at least partly based on the induction of cellcycle arrest, differentiation, and apoptosis (6-9). In addition, in apoptosis-resistant leukemia cell lines and cholangiocarcinoma cell lines, it was shown that autophagy-mediated cell death, i.e., type II programmed cell death, is one of the mechanisms of MK4 inhibiting cell growth $(10,11)$. Autophagy was originally recognized as a biological phenomenon that is enhanced when cells are in starvation and occurs as an adaptation reaction in order for cells to survive $(12,13)$. However, it was reported that exposure to some anticancer agents or radiation also induced autophagy in cancer cells $(14,15)$. Moreover, when 
autophagy induction was inhibited by suppressing the expression of Atg family members involved in autophagy induction with addition of 3-methyladenine (3-MA), a specific inhibitor of class III phosphatidylinositol 3-kinase, or with the RNA interference method, the cell death induced by the exposure to these anticancer agents or radiation was inhibited (14-17). Based on these results, not only apoptosis but also autophagy is believed to be involved in the induction of cell death (18). Nevertheless, while apoptosis has a clear molecular mechanism for cell death induction via activation of caspase or endonuclease, it is not known what molecular basis contributes to the execution of cell death in autophagy. For this reason, it is controversial whether autophagy induction actually causes cell death (autophagic cell death) or whether autophagy occurs in an attempt to escape from cell death (protective autophagy) $(18,19)$.

As described above, the cell growth inhibitory effect of MK4 varies, depending on the type of cancer or cell lines. To the best of our knowledge, there is only one report on detailed examinations of the relationship between colon cancer cell lines and MK4 (20). We thus conducted the present study to evaluate the cell growth inhibitory effect of MK4 using three types of colon cancer cell lines mainly in terms of apoptosis and autophagy.

\section{Materials and methods}

Cell lines and reagents. Three colon carcinoma cell lines, COLO201, DLD-1, and PMCO1 were obtained from the American Type Culture Collection (Rockville, MD, USA). The cells were maintained in continuous culture in RPMI-1640 medium (Gibco, Grand Island, NY, USA) supplemented with 10\% FCS (Hyclone, Logan, UT, USA), 2 mM L-glutamine, penicillin $(50 \mathrm{U} / \mathrm{ml})$, and streptomycin $(100 \mu \mathrm{g} / \mathrm{ml})$. Vitamin K2 (menaquinone-4: MK4) was supplied by Eisai Co., Ltd. (Tokyo, Japan). E-64-d and Pepstatin A, which are inhibitors for lysosomal proteases, were purchased from Biomol International LP (Plymouth Meeting, PA, USA), and 3-methyladenine, an inhibitor of the phosphatidylinositol 3-kinase (PI3K) class III (21), was from Polysciences Inc. (Warrington, PA, USA).

Assessment of viable cell count. After treatment with or without MK4 for various lengths of time, the viable cell numbers were assessed by a WST Cell Counting Kit (Dojin East, Tokyo, Japan), with absorption measurements at $450 \mathrm{~nm}$. In some experiments viable cell counts were assessed by the trypan blue dye exclusion method.

Immunoblotting. Cells were lysed in lysis buffer $(10 \mathrm{mM}$ Tris- $\mathrm{HCl} \mathrm{pH}$ 7.8, $150 \mathrm{mM} \mathrm{NaCl}, 1 \%$ NP-40, 1 mM EDTA, $10 \%$ glycerol, $1 \mathrm{mM}$ phenylmethylsulfonyl fluoride, $0.15 \mathrm{U} / \mathrm{ml}$ aprotinin, $10 \mathrm{mg} / \mathrm{ml}$ leupeptin, $100 \mathrm{mM}$ sodium fluoride, 2 mM sodium orthovanadate), and cellular proteins were quantified using the Protein Assay kit of Bio-Rad (Richmond, CA, USA). Equal amounts of proteins were loaded, separated by SDS-PAGE and transferred onto Immobilon-P membranes (Millipore Corp., Bedford, MA, USA). The membranes were probed with antibodies (Abs) including anti-microtubuleassociated protein 1 light chain 3B (LC3B) Ab, anti-caspase-3
Ab (Cell Signaling Technology, Danvers, MA, USA), anticleaved caspase-3 Ab (Cell Signaling Technology), and antiB-actin Ab (Sigma-Aldrich, St. Louis, MO, USA). Anti-LC3B $\mathrm{Ab}$ was a kind gift from Dr Tomohisa Yokoyama (M.D. Anderson Cancer Center, University of Texas) (22). Immunoreactive proteins were detected by horseradish peroxidaseconjugated second $\mathrm{Ab}$ and an enhanced chemiluminescence reagent (ECR) (Amersham Biosciences Inc., Piscataway, NJ, USA).

Assessment of apoptosis. Apoptosis was detected by morphology and by immunoblotting with either anti-caspase-3 or anticleaved caspase- 3 Abs as described above. For morphologic assessment for apoptosis, the cell suspension was sedimented in a Shandon Cytospin II (Shandon, Pittsburgh, PA, USA), and preparations were stained with May-Grünwald-Giemsa.

Assessment of autophagy. Autophagy was detected by immunoblotting with anti-LC3B in the presence and absence of inhibitors for lysosomal proteases, E-64-d and Pepstatin A, as described above. LC3B protein is known to exist in two cellular forms such as LC3B-I and LC3B-II. LC3B-I is converted to LC3B-II by conjugation to phosphatidylethanolamine, and the amount of LC3B-II is a reliable early marker for the formation of autophagosomes (23-25). Fluorescent immunocytostaining with anti-LC3B Ab and electron microscopy was performed.

Fluorescent immunocytostaining. COLO201 cells treated or not with MK4 were fixed on glass slides by Shandon Cytospin II followed by further fixation with $4 \%$ paraformaldehyde for $20 \mathrm{~min}$. After fixation, the cells on the slides were incubated with anti-LC3B Ab (1:5,000 dilution) in 0.5\% Triton X-100 phosphate-buffered saline (PBS) overnight at $4^{\circ} \mathrm{C}$. The slides were then washed with PBS and incubated with FITC-labeled secondary goat anti-rabbit Ab (1:200 dilution) for $1 \mathrm{~h}$ at room temperature. After washing with PBS, the slides were monitored under a fluorescence microscope BZ-8100 (Keyence, Co., Osaka, Japan).

Electron microscopy. COLO201 cells were treated or not with MK4 and then fixed with a solution containing $3 \%$ glutaraldehyde plus $2 \%$ paraformaldehyde in $0.1 \mathrm{M}$ cacodylate buffer ( $\mathrm{pH}$ 7.3) for $1 \mathrm{~h}$. The samples were further post-fixed in $1 \% \mathrm{OsO}_{4}$ in the same buffer for $1 \mathrm{~h}$, and examined by an electron microscope H-7000 (Hitachi, Tokyo, Japan) as described previously (10). Representative areas were chosen for ultra-thin sectioning and viewed with the electron microscope.

Statistical analysis. The data are given as the mean \pm SD. Statistical analysis was performed using Student's t-test. A p-value of $<0.05$ was considered to indicate a statistically significant difference.

\section{Results}

Growth inhibitory effects of MK4 in colon cancer cell lines. After addition of MK4 at $0.1-100 \mu \mathrm{M}$ to each of the three types of colon cancer cell lines and incubation for $72 \mathrm{~h}$, cell 


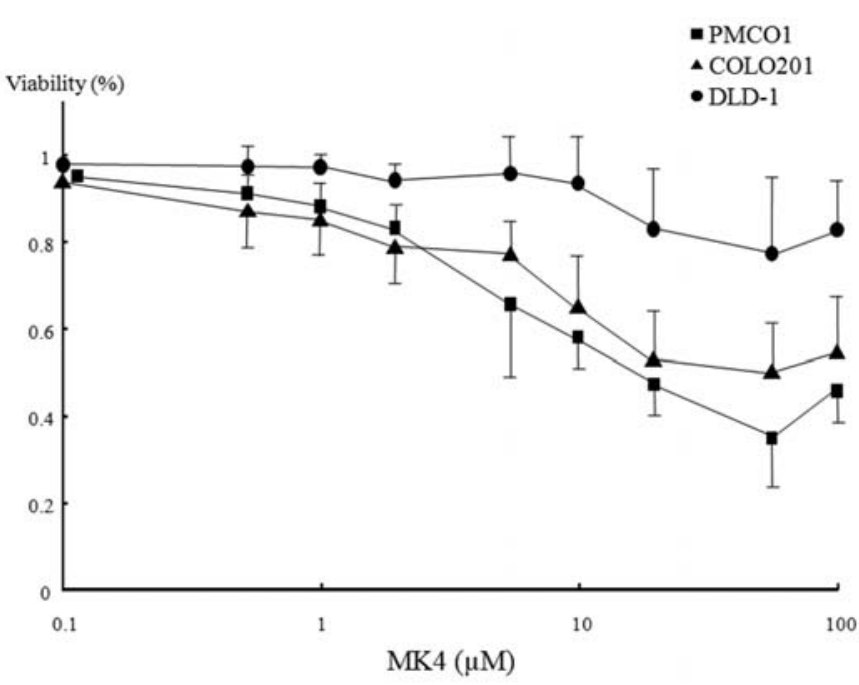

Figure 1. Cell growth inhibitory effect of MK4 in colon cancer cell lines. COLO201, PCMO1 and DLD-1 cells were cultured in the presence of various concentrations of MK4 (0.1-100 $\mu \mathrm{M})$ for $72 \mathrm{~h}$. The number of cells was assessed with the WST cell counting kit as described in Materials and methods. Cell growth is expressed as a ratio to the untreated control cells.

growth inhibitory effects were assessed by a WST assay. Cell growth was inhibited dose-dependently in PMCO1 and COLO201, while the cell growth inhibition observed in DLD-1 was minimal compared with the other two cell lines (Fig. 1).

Morphological features after treatment with MK4 in colon cancer cell lines. Morphological changes in cells at $72 \mathrm{~h}$ after addition of MK4 at a concentration of $50 \mu \mathrm{M}$ were observed after May-Giemsa staining. PMCO1 exhibited typical findings of apoptosis accompanied by condensation of the nuclear chromatin structure and fragmentation of the nucleus (apoptotic bodies). However, no marked morphological changes occurred in COLO201 or DLD-1, but some cells developed cytoplasmic vacuoles. In DLD-1, a portion of cells exhibited apoptotic bodies (Fig. 2).

Immunoblotting with anti-cleaved caspase-3 antibody after treatment with MK4 in colon cancer cell lines. The differences in the induction of apoptosis between the three cell lines as shown in Fig. 2 were determined by an immunoblotting assay with a monoclonal antibody specifically recognizing cleaved caspase-3 after treatment with MK4 in each of the cell lines. As shown in Fig. 3, a band corresponding to cleaved caspase-3 was clearly confirmed in PMCO1. In DLD-1, compared with the control, a slightly intensified band corresponding to cleaved caspase- 3 by the treatment with MK4 was observed. In COLO201, however, no band corresponding to cleaved caspase-3 was observed in the control or MK4treated cell lines, suggesting that the induction of apoptosis after addition of MK4 shown in Fig. 2 correlated well with activation of caspase-3.

Immunoblotting with anti-LC3B antibody after treatment with MK4 in colon cancer cell lines. As shown in Fig. 1, when MK4 was added at concentrations of $10 \mu \mathrm{M}$ or higher, COLO201 exhibited a significant cell growth inhibitory effect, but activation of caspase- 3 did not occur. We previously reported that cell death was induced not via apoptosis but via

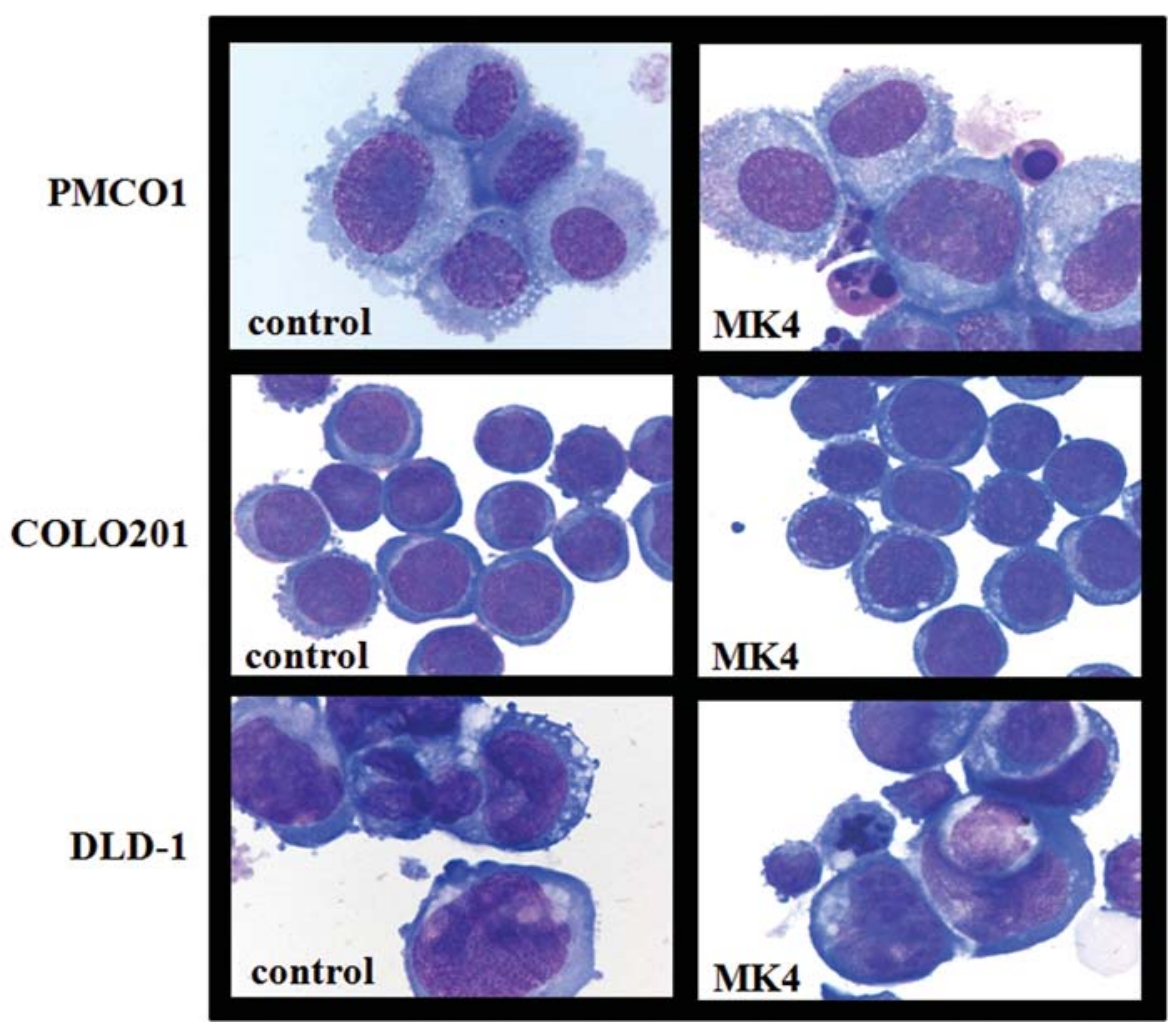

Figure 2. Morphological features after treatment with MK4 in colon cancer cell lines. After 72-h exposure to $50 \mu \mathrm{M}$ MK4, COLO201, PCMO1 and DLD-1 cells were processed for May-Grünwald-Giemsa staining for assessment of morphologic changes as described in Materials and methods (original magnification x1,000). 


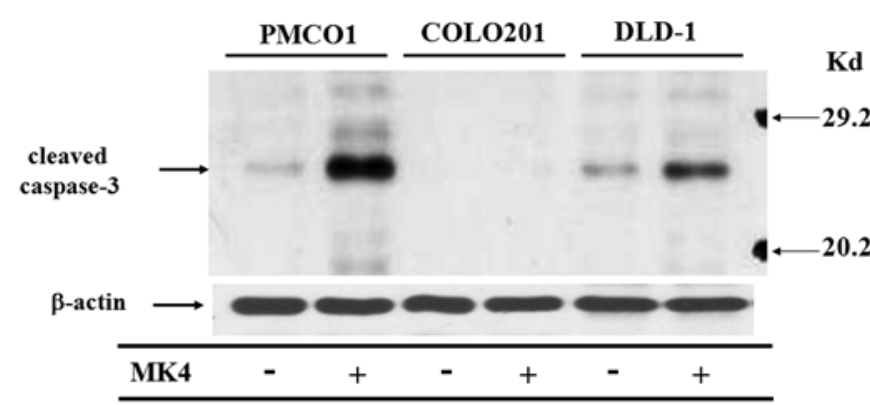

Figure 3. Immunoblotting with anti-cleaved caspase-3 antibody after treatment with MK4 in colon cancer cell lines. Cellular proteins were lysed after 72-h incubation with or without $50 \mu \mathrm{M}$ of MK4. Proteins were separated by either $15 \%$ SDS-PAGE. Aliquots of $40 \mu \mathrm{g}$ of protein extracts were used for immunoblotting using anti-cleaved caspase- 3 and anti- $\beta$-actin Abs .

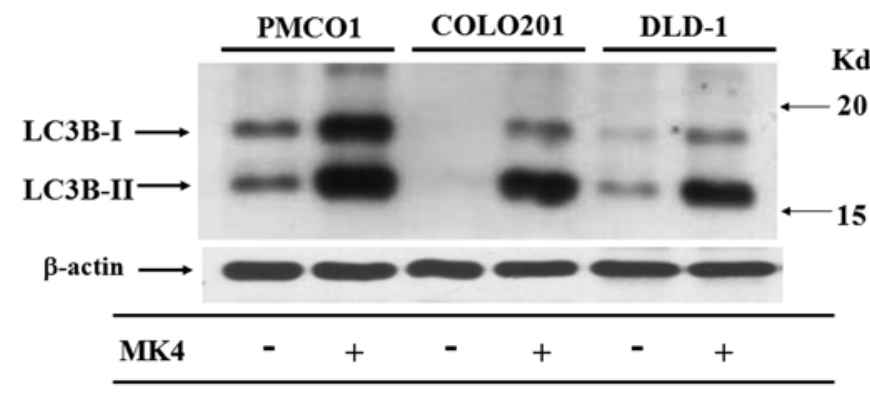

Figure 4. Immunoblotting with anti-LC3B antibody after treatment with MK4 in colon cancer cell lines. Cellular proteins were lysed after 72-h incubation with or without $50 \mu \mathrm{M}$ of MK4. Proteins were separated by either $11.25 \%$ SDS-PAGE. Aliquots of $40 \mu \mathrm{g}$ of protein extracts were used for immunoblotting using anti-LC3B and anti-ß-actin Abs.

autophagy in Bcl-2 overexpressed leukemia cell lines (HL-60bcl-2) when they were exposed to MK4 (10). Based on these results, we performed an immunoblotting assay using an antiLC3B antibody in order to examine autophagy induction after treatment with MK4 in colon cancer cell lines. LC3B undergoes additional modification of phosphatidylethanolamine at an early step of autophagosome formation, thereby resulting in an increased mobility on SDS-PAGE, and is detected as a lower molecular weight band at $15 \mathrm{kDa}$ corresponding to LC3B-II. Evaluation of its expression rate to LC3B-I which has not undergone the additional modification of phosphatidylethanolamine, or the ratio of LC3B-II to LC3B-I, has been used to monitor autophagy induction $(23,24)$. As seen in Fig. 4 , a band corresponding to LC3B-II was expressed at a high level in the three types of cell lines after treatment with MK4.

Expression of isoforms of $L C 3 B$ in the presence and absence of lysosomal protease inhibitors in colon cancer cell lines. The autophagosome fuses with the lysosome to form the autolysosome and is degraded by hydrolyzing enzymes located in the lysosome (25). We therefore compared expression levels of LC3B-II after inhibiting this lysosomal turnover by adding the lysosomal protease inhibitors, E64d and Pepstatin A, to each culture system $6 \mathrm{~h}$ before lysing the cellular proteins. As shown in Fig. 5, although the addition of E64d and Pepstatin A did result in an increased expression level of LC3B-II even

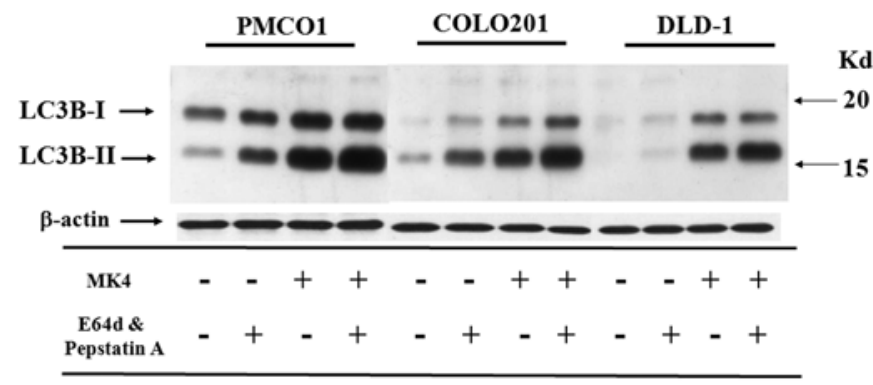

Figure 5. Expression of isoforms of LC3B in the presence and absence of lysosomal protease inhibitors in colon cancer cell lines. After treatment with $50 \mu \mathrm{M}$ of MK4 for $72 \mathrm{~h}$, cells were further cultured with/without protease inhibitors, E-64-d $(10 \mu \mathrm{g} / \mathrm{ml})$ and pepstatin A $(10 \mu \mathrm{g} / \mathrm{ml})$ in the presence of MK4 for $6 \mathrm{~h}$. Cellular proteins were lysed and immunoblotted with anti-LC3B and anti-ß-actin Abs.

before addition of MK4, treatment with MK4 further increased the expression level of LC3B-II in each of the three types of cell lines. These results indicated enhanced autophagy induction by addition of MK4 and incubation in all the three types of cell lines.

Fluorescence immunocytostaining with anti-LC3B antibody in COLO201 cells. To further confirm the involvement of LC3B in MK4-induced autophagy, we performed fluorescence immunocytostaining of COLO201 cells with anti-LC3B Ab. It was previously reported that during amino acid starvation, LC3B becomes localized to form isolated membrane following formation of autophagosome membranes (26). Therefore detection of the punctuated pattern of cytosolic LC3B indicates the involvement of LC3B for autophagosome formation. This phenomenon also has been used as a method to monitor autophagy $(24,27)$. As shown in Fig. 6, untreated COLO201 cells showed diffuse distribution of green fluorescence, whereas treatment with MK4 increased the punctuated pattern of LC3B, representing autophagic vacuoles.

Ultrastructural features of COLO2O1 cells after exposure to $M K 4$. This was further confirmed by electron microscopy of COLO201 cells after 96-h treatment with MK4, showing autophagosomes and autolysosomes in the cytoplasm (Fig. 7B and D). Interestingly, we observed some dying cells without chromatin condensation and apoptotic bodies but showing shrunken nucleus, worn-out cellular membrane and scanty cytoplasms with autophagosomes (Fig. 7C).

Effects of 3-methyladenine on MK4-induced cell growth inhibition in PMCO1 and COLO2O1 cell lines. Since MK4induced cell growth inhibitory effects in both PMCO1 and COLO201 cell lines and also shows autophagy induction (Figs. 1 and 4), we further investigated the possibility of autophagy functions as 'cytoprotective' or 'autophagic cell death' in these cell lines. To inhibit autophagy, we added $2 \mathrm{mM}$ 3-methyladenine, an inhibitor of the class III phosphatidylinositol 3-kinase (21) in the cultures for $72 \mathrm{~h}$ with or without MK4. As shown in Fig. 8, the cell growth inhibition by MK4 was attenuated in COLO201 cells $(\mathrm{p}<0.001 ; 50 \mu \mathrm{M}$ MK4) but not in PMCO1 cells. 


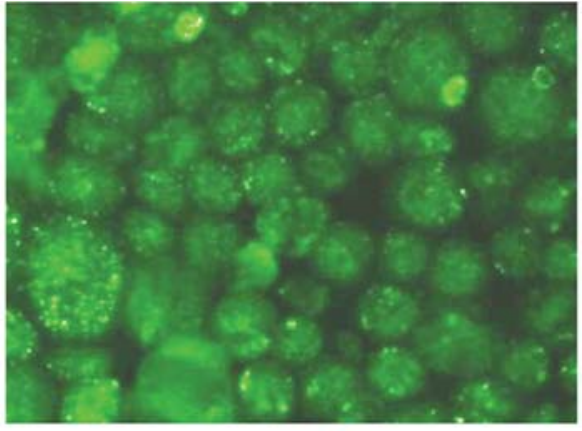

control

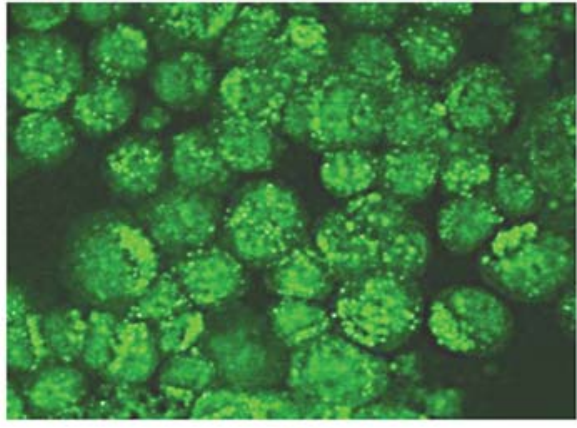

MK4

Figure 6. Fluorescence immunocytostaining with anti-LC3B antibody in COLO201 cells. After exposure to $20 \mu \mathrm{M}$ MK4 for $96 \mathrm{~h}$, COLO201 cells were processed for fluorescence immunocytostaining with anti-LC3B Ab as described in Materials and methods (original magnification x400).
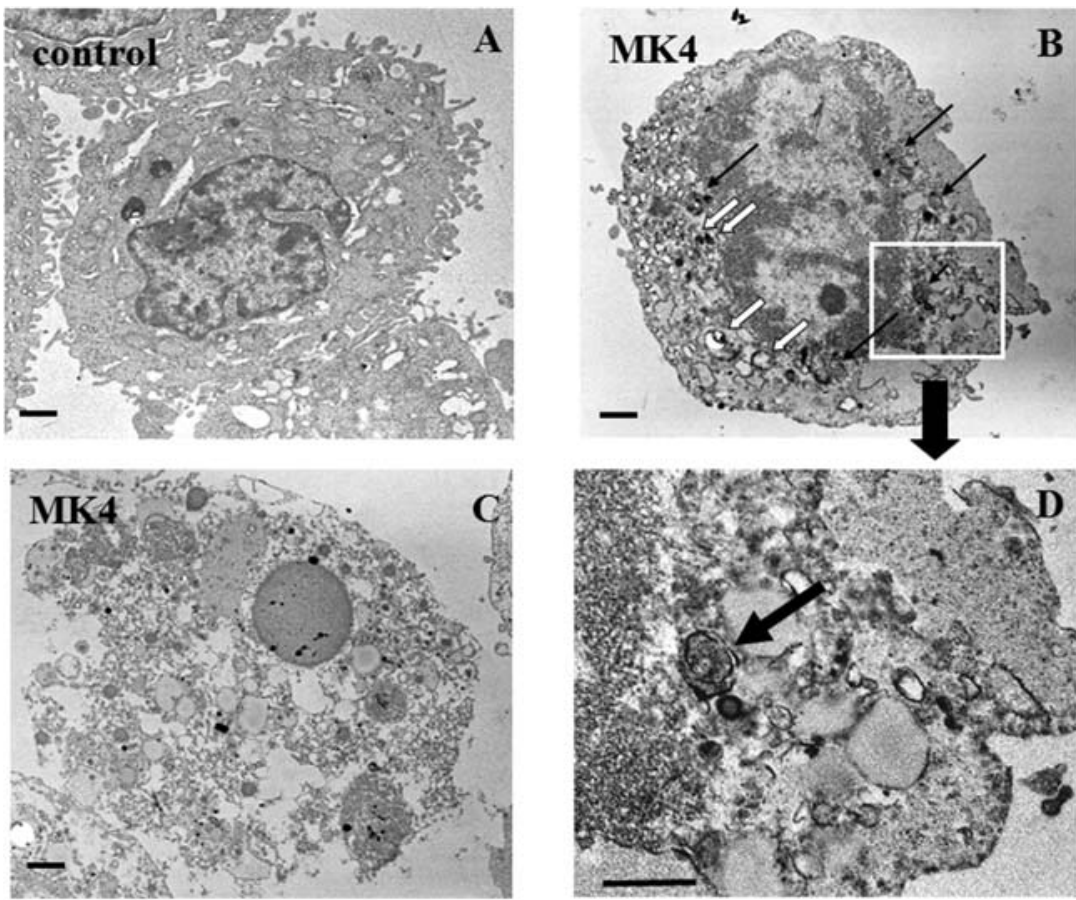

Figure 7. Ultrastructural features of COLO201 cells after exposure to MK4. COLO201 cells treated with or without $50 \mu \mathrm{M}$ of MK4 for $72 \mathrm{~h}$. Then the cells were fixed, and the electron microscopy was performed. Closed arrows indicate autolysosomes and open arrows indicate autophagosomes, respectively.

\section{PMCO1}

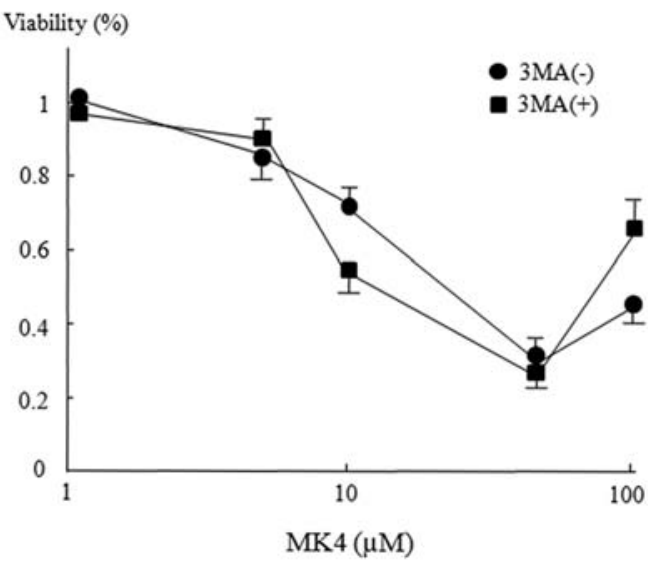

COLO201

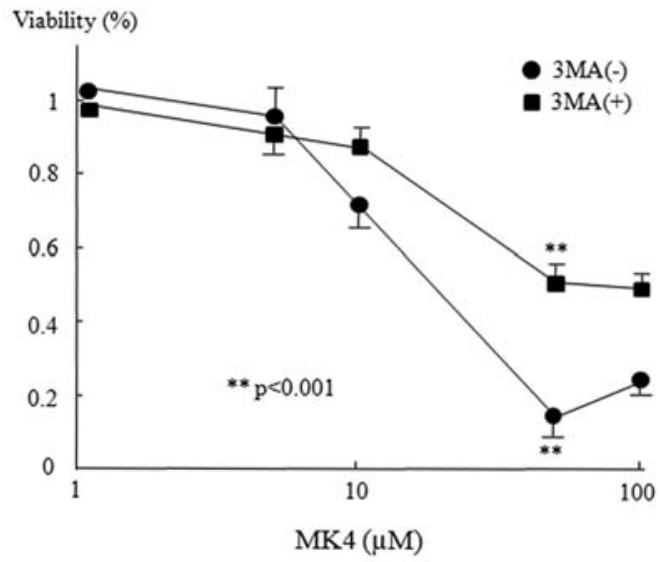

Figure 8. Effects of 3-methyladenine on MK4-induced cell growth inhibition in PMCO-1 and COLO201 cell lines. To inhibit autophagy, 2 mM 3-MA was added to PCMO-1 and COLO201 cultures for $72 \mathrm{~h}$ with or without MK4. The number of cells was assessed with the WST cell counting kit as described in Materials and methods. Cell growth is expressed as a ratio to the untreated control cells. ${ }^{* *} \mathrm{p}<0.001$. 


\section{Discussion}

We set out to determine whether vitamin K2 (MK4) can induce cell growth inhibition in colon cancer cell lines via different types of cell death including autophagy and apoptosis. Our study results demonstrate that addition of MK4 can induce autophagy in all the three types of colon cancer cell (Figs. 4 and 5). MK4 inhibited cell growth dosedependently in two types of cell lines, COLO201 and PMCO1, while the cell growth inhibition observed in DLD-1 was minimal (Fig. 1). In addition, comparison of the two types of cell lines exhibiting growth inhibitory effects showed that apoptosis-mediated cell death was observed in PMCO1 while activation of caspase-3 did not occur in COLO201 (Fig. 3). Moreover, in COLO201, it was suggested that addition of 3-MA resulted in attenuated cytotoxic effect of MK4 and that cell death was induced via autophagy based on the morphological changes on electron microscopy (Figs. 6 and 7). Thus, the biological significance of autophagy induction by MK4 vary in different colon cancer cell lines.

Autophagy is one of the mechanisms inherent in cells for degrading intracellular proteins. This mechanism plays a role in maintaining homeostasis by preventing intracellular accumulation of abnormal proteins and recycling proteins when protein synthesis is excessive or when the nutritional environment becomes poor $(12,28)$. When it comes to most cancers developing in the human body, it is known that cancers that are in a hypoxic state with diminished blood flow are more malignant. When cancer cells are exposed to hypoxic and malnutrition conditions resulting from diminished blood flow, it is known that metabolism may shift from glucose-based energy production to amino acid-based energy production. Generally, a cell under starvation conditions degrades its intracellular proteins and organella, resulting in the induction of autophagy as a response to compensate for deficient amino acid and other nutrition (13). It has been reported that in a certain type of cancer cells under starvation conditions, autophagy is further inhibited and cell death is induced. This indicates that autophagy may contribute to cancer survival under certain conditions. On the contrary, since oxygen and nutrition were present adequately in the cancer cell incubation experiments performed in the present study and the environment was substantially different from that in vivo, there was probably little starvation stress. Therefore, the results of the present study may directly reflect the effects of MK4.

The molecular mechanism of autophagy is divided into three major steps: induction of autophagy, formation of autophagosomes, and degradation in lysosomes (13). The formation of autophagosomes in yeast involves 18 ATG genes. Most of these genes are specific to autophagy and their destructive strains do not express any phenotypes other than those for autophagy deletion. In addition, for most of the ATG genes, their homologous genes are stored even in higher animals and plants $(12,13)$. The Atg proteins are categorized according to their function into the Atg1 protein kinase complex which senses starvation signals, the PI3-kinase complex including Atg6, two covalent bond systems (Atg12 system and Atg8 system), etc., LC3 (Atg8) binds to both the isolation membrane, a precursor of the autophagosome, and the autophagosome membrane; however, it is rarely found in the autolysosome membrane. Although electron microscopic findings are the most reliable method for evaluating autophagy, labeling of autophagosomes using LC3 is also a method for specific observation $(13,24)$. In the present study, an immunoblotting assay using an anti-LC3B antibody showed that a band corresponding to LC3B-II was highly expressed in all three types of cell lines, confirming the induction of autophagy by MK4. In addition, fluorescent immunocytostaining using an anti-LC3B antibody showed that all entire cells exhibited a markedly punctuated pattern, confirming the induction of autophagy (Fig. 5).

Apoptosis, i.e., type I programmed cell death, is a controlled and regulated cell suicide to keep an individual in better condition and it depends on caspase enzymes. This programmed cell death is characterized by morphological changes: cell rounding, condensed nuclear chromatin structure, DNA fragmentation, and cells disruption into small apoptotic vacuoles. Apoptosis is induced via one of the three pathways: i) the death receptor pathway triggered by extracellular signals, ii) the mitochondrial pathway triggered by DNA damage, etc., and iii) the endoplasmic reticulum stress pathway. Eventually, caspase-3 is activated and cell death occurs (29). In a study by Ogawa et al (20) examining the effects of vitamins K2, K3, and K5 using a colon cancer cell line (colon 26), dose-dependent cell growth inhibitory effects of all the vitamins and morphologically typical apoptosis were observed in the cell line, and caspase- 3 was significantly activated compared with the control. In addition, when vitamin $\mathrm{K}$ was administered to mice with colon 26 implanted subcutaneously, a significant growth inhibition and many apoptotic cells were observed compared with the control group (18). In our previous studies, we observed morphologically typical apoptosis in various types of leukemia cell lines including the HL-60 leukemia cell line, a primary culture system of leukemia cells derived from patients with acute leukemia (6), a small cell lung cancer line A547 (8), and a gastric cancer cell line FU97 (9), but we did not note typical findings of apoptosis in any cell lines of the other solid cancer $(9,11)$. Moreover, the percentage of APO2.7 positive cells, an indicator of apoptosis, also showed a significant increase in gastric cancer cell lines other than FU97; however, the percentage tended to increase in three types of cholangiocellular carcinoma cell lines, which was not a statistically significant difference (9). In the present study, we performed an immunoblotting assay using a cleaved caspase- 3 monoclonal antibody to confirm the activation of caspase-3 in PMCO1. Moreover, we observed a slight band corresponding to cleaved caspase-3 in DLD-1, but we did not observe any band in COLO201. PMCO1 also exhibited a morphologically typical picture of apoptosis and this finding was consistent with the observed caspase-3 activity. In COLO201, on the contrary, electron microscopy showed an increased number of cytoplasmic autophagosomes and autolysosomes in some cells, in addition to morphological changes including scantiness of cytoplasm accompanied by loss of cell organelles, nuclear shrinkage, and fragmentation of cytoplasmic membrane in other cells. These findings strongly suggested that autophagic cell death had been induced (Fig. 7C) $(18,30)$. We recently reported that Bcl-2 overexpressed leukemia cell lines (HL-60- 
bcl-2) were resistant to apoptosis induction by MK4, but prolonged incubation resulted in the induction of autophagic cell death. In addition, although autophagy and apoptosis were induced simultaneously by addition of MK4 and incubation in Bcl-2 underexpressed HL-60neo cell lines, it was suggested that cell death via apoptosis occurred first, thereby resulting in masked autophagic cell death (10). Thus, determinants of the cancer cell death phenotypes when cancer cells are exposed to anti-cancer drugs are highly likely to depend on whether the cancer cells are susceptible to the induction of apoptotic cell death. As mentioned above, compared with COLO201, cell growth inhibitory effects are observed in PMCO1 at lower concentrations of MK4, and apoptosis is also induced. On the contrary, apoptosis is not induced by MK4 in COLO201 (Figs. 1 and 3). Furthermore, these findings are consistent with previous reports on the exposure of etoposide to Bax/Bak-double knock-out murine embryonic fibroblasts (17) and the autophagic cell death mainly in a culture system of glioblastoma resistant to anticancer drugs $(15,18)$. Therefore, the induction of 'autophagic cell death' or 'autophagy-mediated cell death' in COLO201 may be due to the acquisition of resistance to apoptosis induction by MK4.

It is speculated that such differences in the final cell response to autophagy induction may reflect differences in cellular factors, e.g., gene expression profiles. In such situations where there are two conflicting concepts, whether the autophagy is 'cytoprotective' or 'autophagic' cell death, is still controversial, we expect that further comparison of gene expression profiles between the three types of colon cancer cell lines may shed light on the variations in biological response to autophagy.

\section{Acknowledgements}

This study was supported by the 'Third-Term Comprehensive Control Research for Cancer' conducted by the Ministry of Health, Labor and Welfare of Japan. The authors are indebted to Professor J. Patrick Barron of the International Medical Communications Center of Tokyo Medical University for his review of this manuscript, and would like to thank Ms. Ayako Hirota and Ms. Minako Suzuki for their expert technical assistance. We also thank Dr Tomohisa Yokoyama of M.D. Anderson Cancer Center, University of Texas for the kind gift of anti-LC3B Ab, and Eisai Co. for providing menaquinone-4.

\section{References}

1. Lamson DW and Plaza SM: The anticancer effects of vitamin K. Altern Med Rev 8: 303-318, 2003.

2. Yaguchi M, Miyazawa K, Otawa M, Ito Y, Kawanishi Y and Toyama K: Vitamin K2 therapy for a patient with myelodysplastic syndrome. Leukemia 13: 144-145, 1999.

3. Miyazawa K, Nishimaki J, Ohyashiki K, Enomoto S, Kuriya S, Fukuda R, Hotta T, Teramura M, Mizoguchi H, Uchiyama T and Omine M: Vitamin K2 therapy for myelodysplastic syndromes (MDS) and post-MDS acute myeloid leukemia: information through a questionnaire survey of multi-center pilot studies in Japan. Leukemia 14: 1156-1157, 2000.

4. Habu D, Shiomi S, Tamori A, Takeda T, Tanaka T, Kubo S and Nishiguchi S: Role of vitamin K2 in the development of hepatocellular carcinoma in women with viral cirrhosis of the liver. JAMA 292: 358-361, 2004.
5. Mizuta T, Ozaki I, Eguchi Y, Yasutake T, Kawazoe S, Fujimoto K and Yamamoto K: The effect of menatetrenone, a vitamin K2 analog, on disease recurrence and survival in patients with hepatocellular carcinoma after curative treatment: a pilot study. Cancer 106: 867-872, 2006.

6. Miyazawa K, Yaguchi M, Funato K, Gotoh A, Kawanishi Y, Nishizawa Y, Yuo A and Ohyashiki K: Apoptosis/differentiationinducing effects of vitamin K2 on HL-60 cells: dichotomous nature of vitamin K2 in leukemia cells. Leukemia 15: 1111-1117, 2001.

7. Yokoyama T, Miyazawa K, Yoshida T and Ohyashiki K: Combination of vitamin K2 plus imatinib mesylate enhances induction of apoptosis in small cell lung cancer cell lines. Int $\mathbf{J}$ Oncol 26: 33-40, 2005.

8. Yoshida T, Miyazawa K, Kasuga I, Yokoyama T, Minemura K, Utsumi K, Aoshima M and Ohyashiki K: Apoptosis induction of vitamin K2 in lung carcinoma cell lines: the possibility of vitamin K2 therapy for lung cancer. Int J Oncol 23: 627-632, 2003.

9. Tokita H, Tsuchida A, Miyazawa K, Ohyashiki K, Katayanagi S, Sudo H, Enomoto M, Takagi Y and Aoki T: Vitamin K2-induced antitumor effects via cell-cycle arrest and apoptosis in gastric cancer cell lines. Int J Mol Med 17: 235-243, 2006.

10. Yokoyama T, Miyazawa K, Naito M, Toyotake J, Tauchi T, Itoh M, You A, Hayashi Y, Georgescu MM, Kondo Y, Kondo S and Ohyashiki K: Vitamin K2 induces autophagy and apoptosis simultaneously in leukemia cells. Autophagy 4: 629-640, 2008.

11. Enomoto M, Tsuchida A, Miyazawa K, Yokoyama T, Kawakita H, Tokita H, Naito M, Itoh M, Ohyashiki K and Aoki T: Vitamin K2-induced cell growth inhibition via autophagy formation in cholangiocellular carcinoma cell lines. Int J Mol Med 20: 801-808, 2007.

12. Mizushima N, Ohsumi Y and Yoshimori T: Autophagosome formation in mammalian cells. Cell Struct Funct 27: 421-429, 2002.

13. Levine B and Klionsky DJ: Development by self-digestion: molecular mechanisms and biological functions of autophagy. Dev Cell 6: 463-477, 2004.

14. Paglin S, Hollister T, Delohery T, Hackett N, McMahill M, Sphicas E, Domingo D and Yahalom J: A novel response of cancer cells to radiation involves autophagy and formation of acidic vesicles. Cancer Res 61: 439-444, 2001.

15. Kanzawa T, Kondo Y, Ito H, Kondo S and Germano I: Induction of autophagic cell death in malignant glioma cells by arsenic trioxide. Cancer Res 63: 2103-2108, 2003.

16. Shao Y, Gao Z, Marks PA and Jiang X: Apoptotic and autophagic cell death induced by histone deacetylase inhibitors. Proc Natl Acad Sci USA 101: 18030-18035, 2004.

17. Shimizu S, Kanaseki T, Mizushima N, Mizuta T, ArakawaKobayashi S, Thompson CB and Tsujimoto Y: Role of Bcl-2 family proteins in a non-apoptotic programmed cell death dependent on autophagy genes. Nat Cell Biol 6: 1221-1228, 2004.

18. Kondo Y, Kanzawa T, Sawaya R and Kondo S: Role of autophagy in cancer development and response to therapy. Nat Rev Cancer 5: 726-734, 2005.

19. Levine B and Yuan J: Autophagy in cell death: an innocent convict? J Clin Invest 115: 2679-2688, 2005.

20. Ogawa M, Nakai S, Deguchi A, Nonomura T, Masaki T, Uchida N, Yoshiji H and Kuriyama S: Vitamins K2, K3 and K5 exert antitumor effects on established colorectal cancer in mice by inducing apoptotic death of tumor cells. Int $\mathrm{J}$ Oncol 31: 323-331, 2007.

21. Selgen PO and Gordon PB: 3-Methyladenine; specific inhibitor of autophagic/lysosomal protein degradation in isolated rat hepatocytes. Proc Natl Acad Sci USA 79: 1889-1892, 1982.

22. Aoki H, Takada Y, Kondo S, Sawata R, Aggarwal R, Aggarwal BB and Kondo Y: Evidence that curcumin suppresses the growth of malignant gliomas in vitro and in vivo through induction of autophagy: role of Akt and extracellular signalregulated kinase signaling pathways. Mol Pharmacol 72: 29-39, 2007.

23. Mizushima N and Yoshimori T: How to interpret LC3 immunoblotting. Autophagy 3: e1-e4, 2007.

24. Klionsky DJ, Abeliovich H, Agostinis P, et al: Guidelines for the use and interpretation of assays for monitoring autophagy in higher eukaryocytes. Autophagy 4: 151-175, 2008.

25. Tanida I, Minematsu-Ikeguchi N, Ueno T and Kominami E: Lysosomal turnover, but not a cellular level, of endogenous LC3 is a marker for autophagy. Autophagy 1: 84-91, 2005. 
26. Kabeya Y, Mizushima N, Ueno T, Yamamoto A, Kirisako T, Noda T, Kominami E, Ohsumi Y and Yoshimori T: LC3, a mammalian homologue of yeast Apg8p, is localized in autophagosome membranes after processing. EMBO J 19: 5720-5728, 2000.

27. Bampton ETW, Goemans CG, Niranjan D, Mizushima N and Tolkovsky AM: The dynamics of autophagy visualized in live cells. Autophagy 1: 23-56, 2005.
28. Klionsky DJ and Emr SD: Autophagy as a regulated pathway of cellular degradation. Science 290: 1717-1721, 2000

29. Green DR and Reed JC: Mitochondria and apoptosis. Science 281: 1309-1312, 1998

30. Tsujimoto Y and Shimizu S: Another way to die: autophagic programmed cell death. Cell Death Differ 12: 1528-1534, 2005. 